\title{
Simplified Guide to MR Elastography in Early Detection of Hepatic Fibrosis with Case Reports
}

\section{The New Norm in Assessing Liver Health}

\author{
Amit Kharat ${ }^{1}$ Nikhith Soman Vanpully ${ }^{1}$ Jacob Cheeran Jeeson ${ }^{1}$ \\ ${ }^{1}$ Department of Radiology, Dr. D.Y. Patil Medical College, Hospital \& \\ Research Centre, Pimpri, Pune, Maharashtra, India \\ Address for correspondence Nikhith Soman Vanpully, MD, \\ Department of Radiology, Dr. D.Y. Patil Medical College, Hospital \& \\ Indian J Radiol Imaging 2021;31:644-652. \\ Research Centre, Pimpri, Pune, Maharashtra 411018, India \\ (e-mail: nikhithsoman@gmail.com).
}

\begin{abstract}
The current unhealthy diets and sedentary lifestyle have led to increase in the prevalence of diabetes and metabolic syndrome globally. Fatty liver is a common occurrence in metabolic syndrome. The liver health is often ignored due to delayed warning signs. Fatty changes of the liver is one of the common findings in ultrasonography. Ultrasound does not detect fibrosis except when cirrhosis is developed. Early stages of fibrosis are asymptomatic with no significant laboratory or preliminary imaging findings. With fibrosis, the elasticity of the liver is reduced and becomes stiffer. Over the years, many techniques have developed to assess the stiffness of the liver, starting from palpation, ultrasonography, and recently developed magnetic resonance elastography (MRE). In this article, we have tried to simplify the concepts of MRE to detect fibrosis and present few case reports. The basic steps involved in generating elastograms and interpretation with some insight on how to incorporate it into the clinical

Keywords

- elastography

- magnetic resonance elastography

- liver stiffness

- elastograms workflow are discussed. MRE is superior to various other available techniques and even offers certain advantages over biopsy. MRE is FDA approved for liver fibrosis since 2009, yet it is hardly used in the Indian setting. MRE is a safe and noninvasive technique to evaluate a large volume of the liver and can be a new norm for the evaluation of fatty liver. Magnetic resonance imaging (MRI)-based elastography techniques hold an exciting future in providing mechanical properties of tissues in various organs like spleen, brain, kidney, and heart.
\end{abstract}

Affiliated institute-Dr. D.Y. Patil Medical College, Hospital E Research Centre, Pimpri, Pune. It is one of the first medical colleges to provide MRI-based liver elastography in the country and since its introduction in 2018, Dr. Amit Kharat has been actively involved in setting the protocol and reporting formats for the same. We have done over 100 plus MRE studies since its inception. This review focuses on simplifying the concepts involved and how to incorporate it into day-to-day practice.

Addition information-This is our second time submission of the same article to IJRI (old ID - IJRI_517_20), it was provisionally accepted after the double peer review process, corrections; it also completed the technical and language check in January of 2021. But since the change in publication houses there was no acknowledgment of the status of the article. I was asked to resubmit after multiple enquiries. Please do the needful as the article has been in the review process for almost a year.

DOI https://doi.org/ 10.1055/s-0041-1735929. ISSN 0971-3026.

\section{Introduction}

The spectrum of diseases causing morbidity has dramatically changed with the turn of the century from infectious to that of lifestyle based. Unhealthy diet and sedentary lifestyle have led to increase prevalence of diabetes and metabolic syndrome, ${ }^{1}$ but the consequential liver involvement is often ignored due to delayed warning signs, lack of safe and easy options for better evaluation. In the daily routine of a sonologist at least 20 to $30 \%$ of patients walk out with a report stating fatty changes of the liver, seen across the population be it alcoholic, nonalcoholic, women, young, and old. ${ }^{1}$ But the problem is they are limited to having their liver enzymes tested or mostly just ignored alike

(c) 2021. Indian Radiological Association. All rights reserved.

This is an open access article published by Thieme under the terms of the Creative Commons Attribution-NonDerivative-NonCommercial-License, permitting copying and reproduction so long as the original work is given appropriate credit. Contents may not be used for commercial purposes, or adapted, remixed, transformed or built upon. (https://creativecommons.org/ licenses/by-nc-nd/4.0/)

Thieme Medical and Scientific Publishers Pvt. Ltd., A-12, 2nd Floor, Sector 2, Noida-201301 UP, India 
by patients and physicians. The fatality that we are talking about is the end stage of cirrhosis which is a silent killer, exactly showing up when there is no point of return. It is currently the 11th most common cause of death globally, with more than 2 billion people consuming alcohol worldwide, a similar number of obese and overweight adults and over 400 million with diabetes, the importance of liver health is more crucial than ever before. ${ }^{2}$ With the cutting edge advances in magnetic resonance imaging (MRI), it is currently possible to accurately assess the composition and physical stiffness of the liver using the principle of propagation of waves and gradientbased MRI sequences. ${ }^{3}$

A normal liver is soft and demonstrates elasticity. On repeated insults by chronic inflammation due to multiple factors its stiffness keeps increasing, due to developing fibrosis and if the process continues, inevitably reaches cirrhosis and later hepatocellular carcinoma (HCC). In each case scenario the stiffness keeps increasing and a tool to quantify this mechanical property of tissue will play a crucial role in early detection, grading and assessment of treatment response. ${ }^{4}$

In this article, we try to simplify the complex processes involved in MRI and enforce its role in justifying day to day clinical practice. The latest MRI systems offer liver packages with the ability to quantitatively assess the mechanical properties of the liver along with add-ons like volumetric estimation of fat and iron. These act as a tool to detect the dormant phase of reversible liver fibrosis, hence, enabling appropriate management.

In MRI-based elastography of the liver, we cannot only diagnose but also stage the degree of fibrosis by sampling a large volume of the liver in an objective manner with high repeatability, providing data that is comparable with histology reports. This can provide a greater understanding of liver health and needs to be a new norm in assessing liver health especially in risk groups, considering the large population affected by liver diseases. ${ }^{5}$

\section{Liver Stiffness and the Progression of Fatty Liver Disease}

The most common causes of fatty liver consist of alcoholism and metabolic syndrome. Chronic alcoholic abuse as we know affects the liver via multiple pathways including direct toxicity and fat accumulation. While metabolic causes such as obesity, diabetes, and dyslipidemia can also cause fatty liver, its accumulation can cause repeated injury to the hepatocytes (-Fig. 1). Simple fatty liver or hepatic steatosis as such does not lead to fibrosis or increase in liver stiffness, but once inflammation sets in depending on genetic as well as environmental factors the process of fibrosis slowly starts. A series of repeated injury and inflammation inevitably leads to cirrhosis and in some cases HCC ( - Fig. 1). Various studies suggest that with $1 \mathrm{kPa}$ increase in liver stiffness the risk of HCC increases by more than $3 \%$. Hence, the main aim is to detect these early changes in liver stiffness indicating fibrosis, as well as to assess the progression of the disease. ${ }^{6,7}$

\section{Basics of Elastography}

Elastography, if simply described, is a "sophisticated palpation technique" or "palpation by imaging," a method of evaluating stiffness of tissue. ${ }^{8}$ Earlier, stiffness was assessed subjectively using techniques of palpation; with the advancement in imaging techniques, newer methods have evolved. There are various types of elastography based on the principle applied and imaging modality used, as demonstrated in -Fig. 2. Mainly two principles are used in imagingbased elastographic techniques, static and dynamic. In static/strain imaging, the change in shape or deforming ability of the affected tissue is assessed in comparison with the normal area by the application of manual compression (-Fig. 3). This is especially useful in evaluating superficial tissue such as muscle and breast. However, its role in assessing deeper organs like the liver is poorer, especially in detecting smaller changes in stiffness (stage F1 and F2 of fibrosis). The more popular and sensitive techniques are based on the principle of waves propagation. ${ }^{3}$ The velocity of waves through the tissue is assessed and stiffness is calculated (-Fig. 3). The waves can be acoustic-based like in acoustic radiation forced impulse (ARFI) or vibrationbased like in Fibroscan and magnetic resonance elastography (MRE). ${ }^{9}$

\section{Principle of MRI Elastography}

Here we use the properties of mechanical waves to assess the stiffness of the tissue. We know that propagating waves move slowest in air, faster in liquid, and fastest in solids, in other words, stiffer the tissue faster the propagation (- Figs. 3 and 4). This simple principle is used and combined with MRI-based imaging sequences to capture the waves and
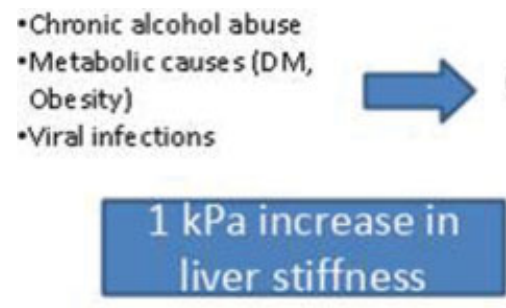

\section{Chronic inflammation
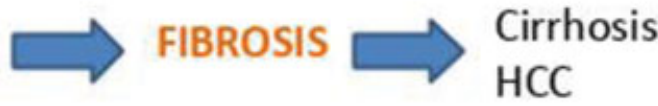

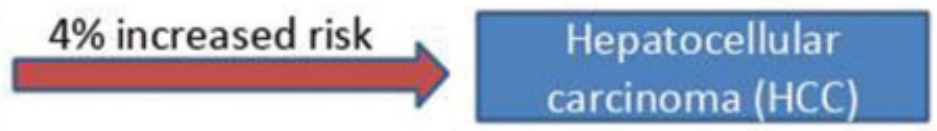

Fig. 1 Flowchart shows the progression of liver disease and increased risk of hepatocellular carcinoma (HCC). 


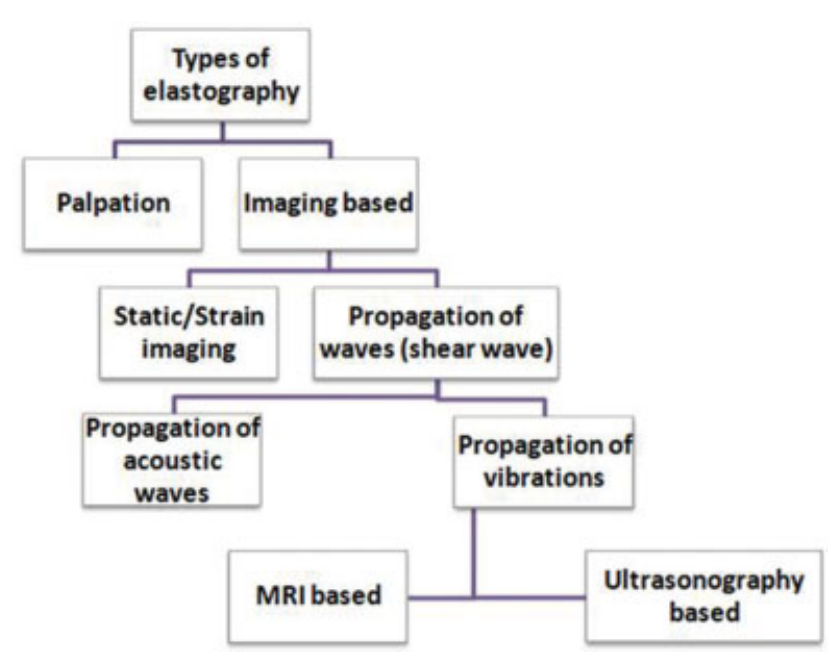

Fig. 2 Types of elastography techniques used classified based on principle and imaging modality.

using algorithms to calculate the stiffness in kilopascals in any given location in the liver. There are basically three steps involved in the process of acquiring the final images called elastograms.

\section{Step 1-Production of Shear Waves}

To vibrate the organ in a controlled manner a system of active and passive drivers is used. The active driver is the machine that produces the vibrations and is placed outside the MRI room. Using an MRI compatible tube, the vibrations are transmitted to a passive driver, with frequencies ranging between 50 and $500 \mathrm{~Hz}$. The passive driver is placed over the liver. There are a couple of types of passive drivers currently available: rigid and flexible type. The flexible type can conform around the chest and can produce better wave propagation as shown in the image (-Fig. 4).

\section{Step 2-Capturing the Propagation of Waves}

The mechanical waves propagating through the organ are captured using various sequences with added motion encoded gradients (MEGs). The vibrations generated are synchronized with the MEGs to sensitize the sequences to the cyclic motion of the spin to generate images to assess the tissue displacement (-Fig. 5). The main basic sequences used currently are gradient echo (GRE), echoplanar imaging, and spin-echo sequences. ${ }^{3,10}$ The resultant wave images are evaluated on the basis of its luminosity, brighter the image better the wave propagation forming reliable stiffness maps. Hence, these images also play an important role in quality control.

\section{Step 3-Applying Inversion Algorithms to Process Elastograms}

The wave images acquired are assessed on the basis of tissue displacement, using complex inversion algorithms. The final images generated are called elastograms, these are color code images showing various grades of stiffness. The colors range from purple to red with increasing stiffness. The values are taken in $\mathrm{kPa}$ and graded according to various systems (-Fig. 6). ${ }^{3,10}$ Further, some systems apply grids over these elastograms using confidence algorithms to mark out the liver to obtain the most reliable value; these images are called confidence maps.

\section{Performing the Scan}

The patient is asked to come in a fasting state of at least 4 hours, then made to lie supine on the table, after which a disk-shaped driver with a connected tube is placed over the lower chest wall on the right side at the midclavicular line just over the position of the liver corresponding to the lower end of sternum; it is harnessed tightly with a VELCRO and then finally the body torso coil is placed over it. ${ }^{11}$ Once the imaging starts the driver begins vibrating, transmitting the vibrations to the liver; then using MR-based motion encoded sequences, cross-sectional images are generated. Currently, GRE-based MRI sequences are used, newer better acquisition using spin-echo sequences is also available. ${ }^{11}$ Axial images are captured by at least four slices through the widest crosssectional area of the liver. Each slice takes around 15 seconds for acquisition, considering time for patient positioning and sequence planning, a total running time of approximately 10 minutes is sufficient for the whole process. a
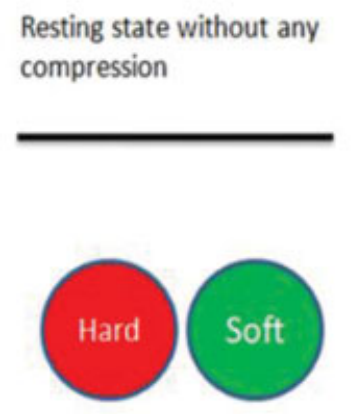

Manual compression
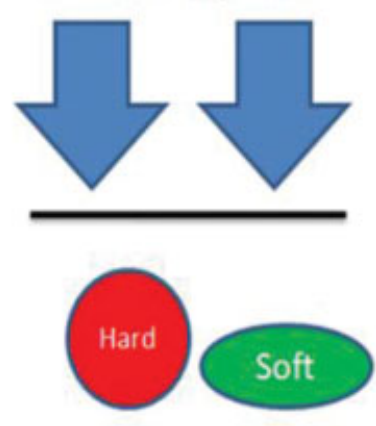

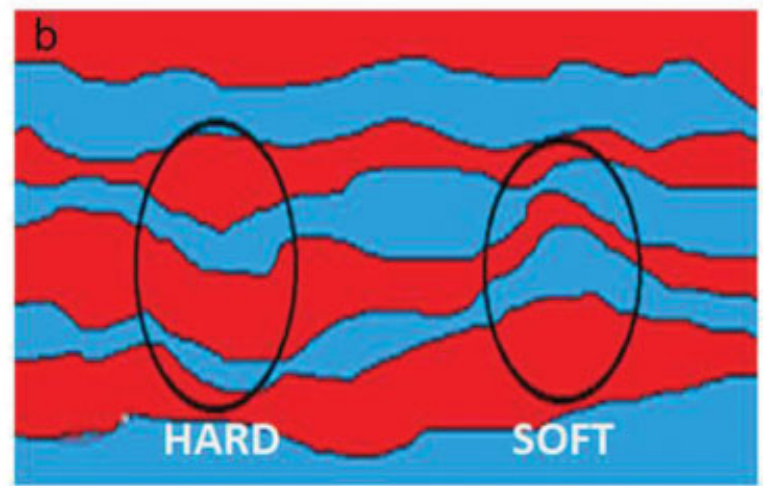

Fig. 3 These are schematic diagrams showing the difference between static and dynamic elastography. The figure on the right (a) shows how soft and hard tissues deform on application of manual pressure; this difference is assessed in static/strain elastography. The image on the left (b) shows how propagating waves speed up as they pass through harder tissue as compared to soft tissue, it better shown in $\mathbf{- F i g . ~} \mathbf{4}$. 


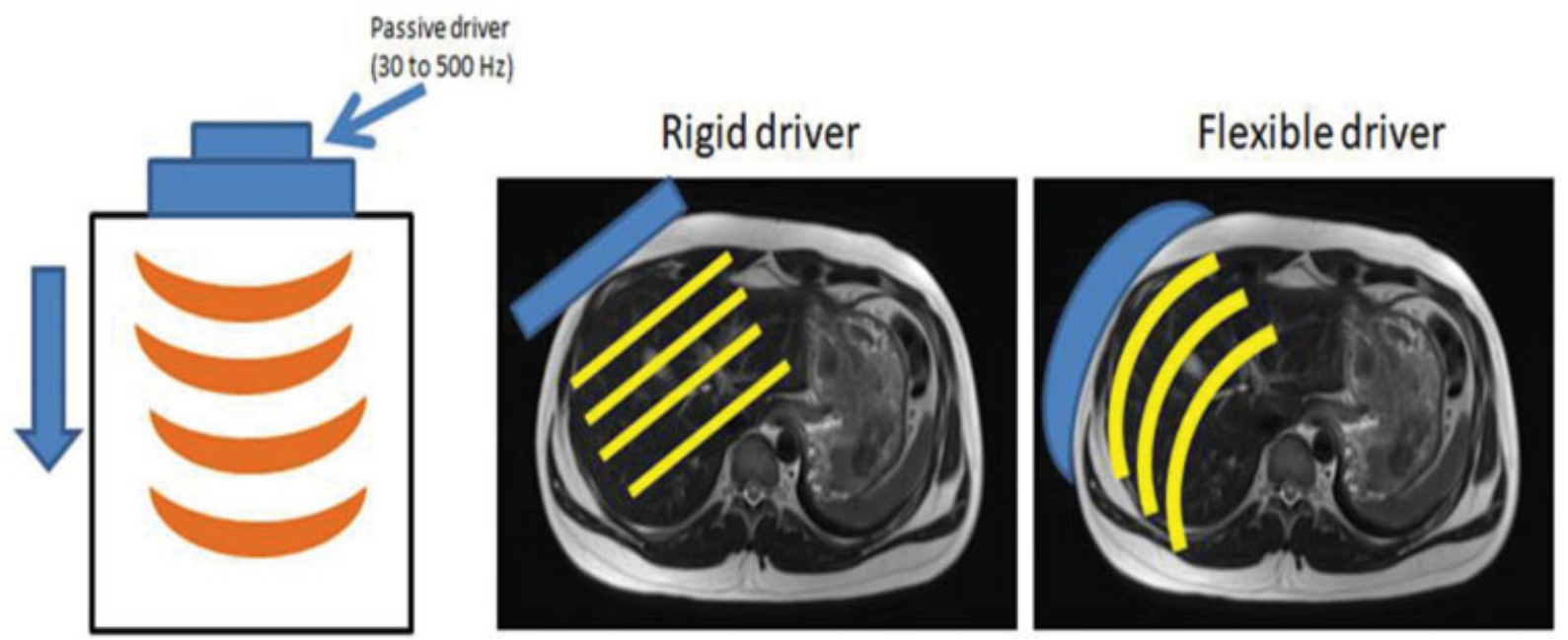

Fig. 4 Step one is producing the mechanical waves using a vibrating passive driver at a frequency of $30 \mathrm{Ho} 500 \mathrm{~Hz}$. The types of drivers used can be rigid or flexible type with the latter producing better wave propagation.

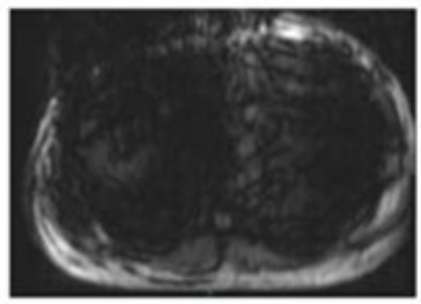

Magnitude image

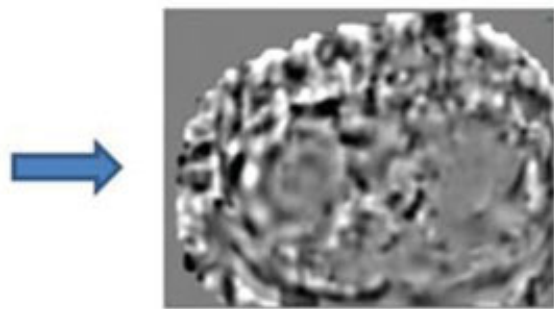

Phase image

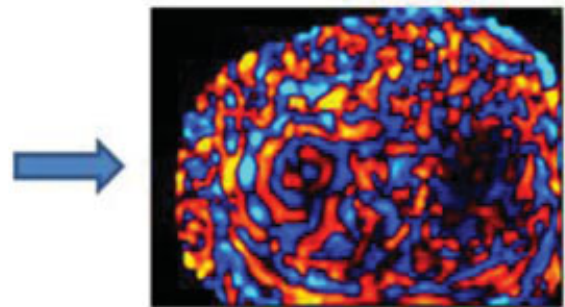

Colour wave image

Fig. 5 Step two is capturing wave images. These magnitude, phase, and wave images are generated sequentially and obtained using motionencoded sequences for image acquisition. These images need to be assessed for quality control.

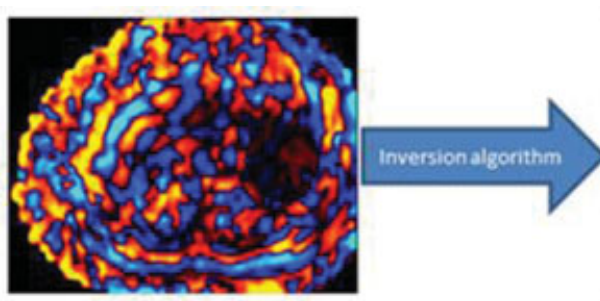

Wave image

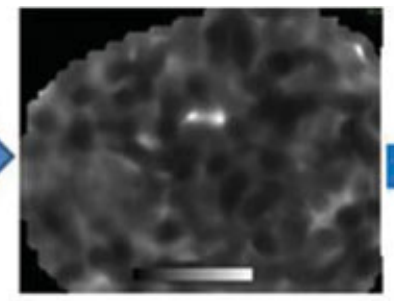

Grey scale stiffness map

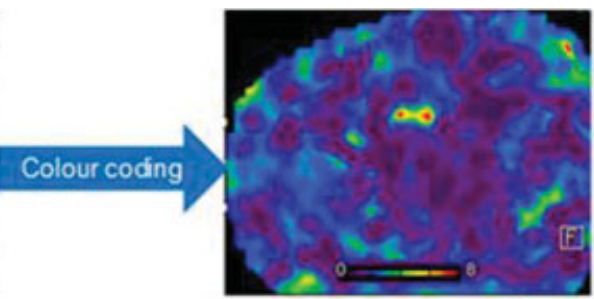

Elastogram

Fig. 6 Step three is applying inversion algorithms on the acquired wave images to assess tissue displacement and finally generate elastograms.

\section{Interpretation of Data}

The images generated prior to the elastogram are important for quality assessment. The magnitude image is evaluated for adequate vibrations generated by the passive driver, seen as signal drop of the subcutaneous fat just beneath the driver. The phase and color-coded images should be assessed for adequate propagation of waves through the liver, higher the illumination of the wave images, better the propagation. ${ }^{12}$

The final image generated is called elastograms which are color-graded cross-sectional images; in some systems the liver is outlined using grids. The elastograms can provide specific stiffness values in kilopascals in any specified region of interest (ROI). ${ }^{10}$ The stiffness values from various ROIs are then taken and graded on the basis of their stiffness. The ROIs can be drawn as multiple rounds to oval areas or a large single geographical pattern in each axial slice. But it must be noted to avoid areas of large vessels, gall bladder, artefacts, some vendors provide stiffness maps excluding such areas with grids (-Fig. 8). ${ }^{12}$

The grading of fibrosis can be done in comparison to various studies showing the stiffness of the liver and its 


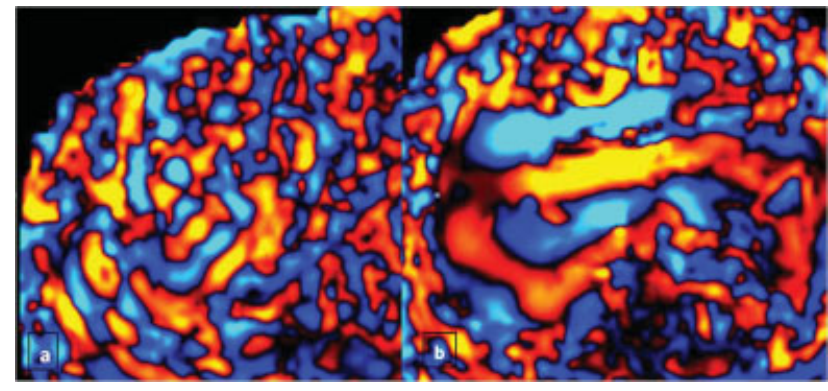

Fig. 7 These are wave images acquired using the 3-Tesla MRI in two different patients. Panel (a) shows a normal liver and panel (b) shows a liver with increased stiffness, the wavelength is significantly increased in (b) based on the principle that propagating waves move faster through stiff tissue.

histopathological grading. The main pathological gradings compared to are METAVIR and Batts-Ludwig. Some of the studies with larger sample size and their results of cut off in different grades are compared in - Table 1. We have considered only studies that are graded on the basis of METAVIR classification. In these studies, the stiffness values obtained were compared to their fibrosis grading on histopathology. ${ }^{13}$ Elastograms with various stiffness values are shown in -Fig. 8. In the cases discussed the cut off for fibrosis grading was set according to Yin at el. ${ }^{14}$

\section{Role of MR Elastography with Case Reports}

In our practice, we have had scenarios where the patients were completely asymptomatic and mostly underwent the procedure as a part of their regular health check-up.

Case 1: A 68-year-old businessman came to our hospital for a general check-up, with a history of Type II DM for 20 years, no history of alcoholism. On examination, there were no significant findings. His laboratory reports showed controlled blood sugar levels with normal LFTs as well as other blood parameters. He had some nonspecific abdominal complaints following which ultrasonography was done and the report was normal with liver showing normal echogenicity. He also underwent an endoscopy, and it incidentally showed few prominent varices in the lower esophagus, sparking suspicion of portal hypertension. Hence, he was
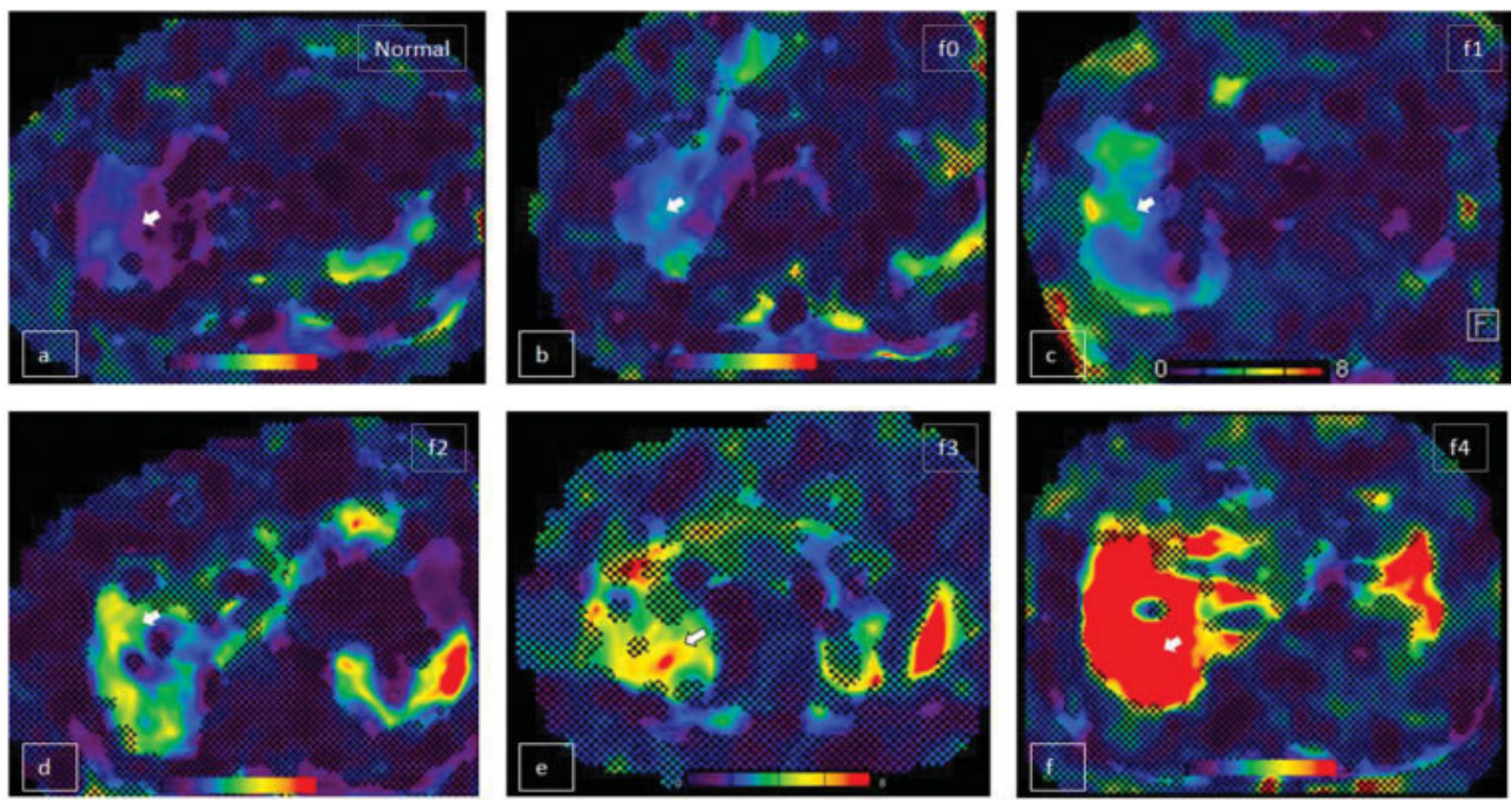

Fig. 8 These are elastograms acquired on 3 Tesla MRI showing liver stiffness in six different patients demonstrating different grades of liver stiffness. The mean stiffness values were (a) $1.5 \mathrm{kPa}$ in normal, (b) $2.6 \mathrm{kPa}$ in F0, (c) $3.8 \mathrm{kPa}$ in F1, (d) $4.5 \mathrm{kPa}$ in F2, (e) $5.2 \mathrm{kPa}$ in F3, and (f) $8.7 \mathrm{kPa}$ in F4 as labeled (white arrow) on the images. The color coding changes from purple to red corresponding to increased stiffness.

Table 1 Fibrosis grading in various studies and their cutoffs in $\mathrm{kPa}$

\begin{tabular}{|c|c|c|c|c|c|c|c|c|}
\hline Study & year & Sample size & Classification used & F0 & F1 & $\mathrm{F} 2$ & F3 & F4 \\
\hline Yin at el ${ }^{14}$ & 2007 & 48 & METAVIR, Batts-Ludwig, Brunt & 3.79 & 4.01 & 5.22 & 6.01 & 8.21 \\
\hline Wang et $\mathrm{al}^{15}$ & 2011 & 71 & METAVIR, Batts-Ludwig, Brunt & 3.33 & 3.90 & 5.43 & 7.70 & 8.87 \\
\hline Rustogi et al ${ }^{16}$ & 2012 & 76 & METAVIR, Batts-Ludwig, Brunt & 3.19 & 4.52 & 4.64 & 7.29 & 7.62 \\
\hline Low and Hassanein ${ }^{17}$ & 2012 & 101 & METAVIR & 2.35 & 3.92 & 4.34 & 6.12 & 8.06 \\
\hline Venkatesh et al ${ }^{18}$ & 2014 & 63 & METAVIR & 2.52 & 2.88 & 3.49 & 4.35 & 6.54 \\
\hline Ichikawa et al ${ }^{19}$ & 2012 & 114 & METAVIR & 2.10 & 2.42 & 3.16 & 4.22 & 6.21 \\
\hline
\end{tabular}


advised MR elastography of the liver as a noninvasive test, instead of biopsy. On MR elastography, the liver showed an extensive increase in stiffness involving the whole liver with mean stiffness value of $8.9 \mathrm{kPa}$ (-Fig. 9) and on follow-up biopsy it showed grade 44 fibrosis (METVIR). Most of the liver diseases are silent until it reaches a late stage, hence using MRE as a screening tool in patients with risk factors for the development of cirrhosis can be crucial.

Case 2: In another scenario, a male in his mid-thirties came for the test following concerns about his liver health due to his unhealthy lifestyle for many years. His ultrasonography showed grade 2 fatty liver with hepatomegaly. His laboratory profile was well within normal limits. On MRE there were early changes of increased stiffness all over the liver with mean value of $2.6 \mathrm{kPa}$ ( - Fig. 10). Since it was a very early stage, he was advised some lifestyle modifications and cautioned about the possibility of development of cirrhosis in a later stage. He was also suggested follow-up scans to evaluate treatment response. In this case, the patient was sensitized to the changes in liver stiffness and forced him to willfully undergo changes in his lifestyle.

It can especially play an important role in patients with chronic hepatitis, especially type $C$, since many advanced treatment modalities are now available. Hence the grading of fibrosis is crucial for the appropriate management; followup scans will also play a vital role to assess treatment response which would have required liver biopsy previously, but with MRE it can be done safely and instantly providing the data of a large sample of the liver. In other scenarios, some patients with chronic medication, for example, severe psoriasis on methotrexate, required to undergo yearly liver biopsy for detecting fibrosis, it can be completely replaced by MRE providing much safer and accurate insight. ${ }^{20}$

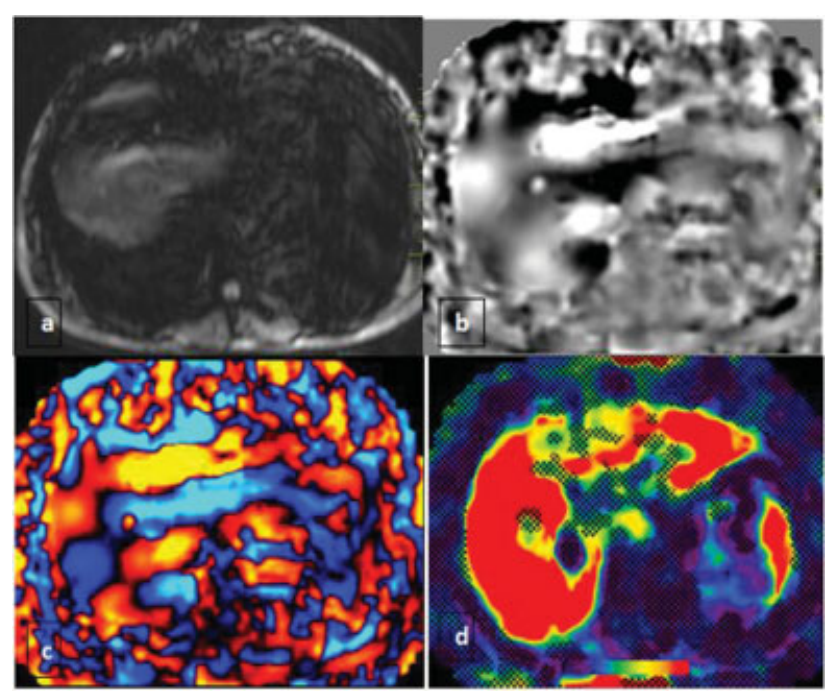

Fig. 9 A 68-year-old male came for a general check-up, with a history of type II DM for 20 years, with no history of alcoholism. These are magnitude (a), phase (b), wave (c), and elastogram (d) images obtained using the 3-Tesla MRE showing significantly increased stiffness, with mean liver stiffness measurement of $8.9 \mathrm{kPa}$.

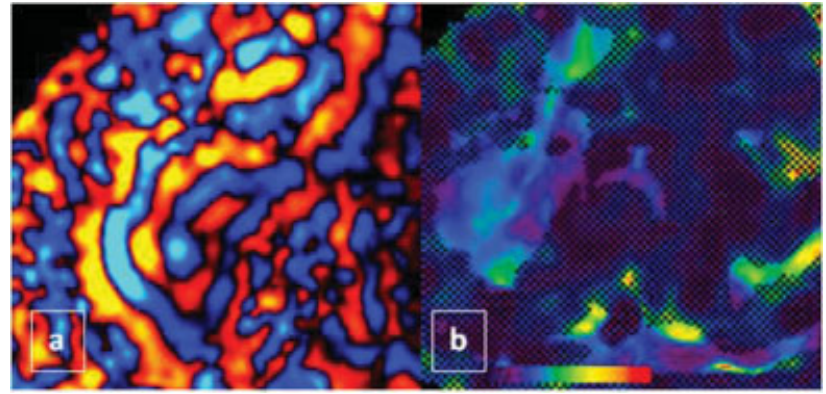

Fig. 10 These are wave (a) and elastogram (b) images obtained using the 3-Tesla MRI showing mildly increased stiffness with a mean value of $2.6 \mathrm{kPa}$ suggesting grade F0 of fibrosis.

\section{Workflow for Patient Screening}

The absolute edge of elastography is in detecting the change in liver stiffness in the very early stage of fibrosis; this can be effectively made use of only if it is done as a screening tool. It is not only good for detecting fibrosis but can also accurately quantify the stiffness and grade the fibrosis. So, who should undertake the test? We can divide the possible candidates into two, one those who have positive liver findings on ultrasound, like that of raised echogenicity or heterogeneous echotexture, other should be persons with risk factors like obesity, alcoholics, hepatitis, metabolic syndromes, and altered liver enzymes. These patients are ideal to undergo the test to provide a wholesome evaluation of liver health. Detecting fibrosis in the early stages can be crucial since it can be reversible and also more importantly sensitize the patients about the same. ${ }^{14}$ Most patients after realizing that they have a high risk of going into cirrhosis voluntarily make huge changes in their lifestyle.

\section{Limitations of Ultrasound-Based Techniques}

Ultrasound-based techniques are definitely more affordable and easy to use, but it comes with lots of drawbacks. Firstly, it provides data of a very limited area of the liver at a time and often becomes cumbersome to evaluate the whole liver (-Table 2). It can lead to missing areas of focal fibrosis. Effective evaluation is often limited by body habitus be it obesity, intervening ribs, or interpolated bowel. These methods are often very dependent on the operator and large interobserver variability is noted. In many cases, it is just qualitative and specific stiffness values are difficult to attain. $^{2}$

\section{Technological Superiority of MR Elastography}

MRI has already established itself as a gold standard in soft tissue imaging, combining that with properties of propagation of mechanical waves through the liver and we have a tool that can provide information at a more advanced level. A biopsy is considered the gold standard in detecting fibrosis, but it is accompanied by a lot of limitations, firstly sampling error, the sample assessed is 
Table 2 Comparison of currently used elastography technique

\begin{tabular}{|l|l|l|l|l|l|}
\hline Type & Cost & Accuracy & $\begin{array}{l}\text { Sample } \\
\text { size }\end{array}$ & Advantage & Limitation \\
\hline $\begin{array}{l}\text { Static/Strain } \\
\text { elastography/ARFI }\end{array}$ & Low & $\begin{array}{l}\text { Low, less sensitive } \\
\text { to lower grades } \\
\text { of fibrosis }\end{array}$ & Small & $\begin{array}{l}\text { Available in most } \\
\text { USG machines }\end{array}$ & $\begin{array}{l}\text { Body habitus, interob- } \\
\text { server variation, poor } \\
\text { sensitive, ascites }\end{array}$ \\
\hline $\begin{array}{l}\text { Transient elastography/ } \\
\text { Fibroscan }\end{array}$ & Low & High & Small & Accurate and cheap & Body habitus, ascites \\
\hline MRE & High & High & Large & $\begin{array}{l}\text { Not limited by body habitus, } \\
\text { no inter-observer bias. }\end{array}$ & $\begin{array}{l}\text { Iron deposit and } \\
\text { ascites }\end{array}$ \\
\hline
\end{tabular}

Abbreviations: ARFI, acoustic radiation forced impulse; MRE, magnetic resonance elastography; USG, ultrasonography.

very small, about $1 / 10,000$ the volume of liver, if a sample is taken from the wrong area even high-grade fibrosis will appear as normal or vice versa. It is also often associated with high interobserver variability. MRE can in fact aid biopsy in a huge way by providing the exact areas of increased stiffness for procuring better samples. Biopsies are painful and uncomfortable procedures while also related to mortality and morbidity. ${ }^{21}$ Compared to the ultrasound-based techniques, instantly we have access to the whole liver without any limitations of body habitus. There is a limited role of interobserver bias as the data is completely autogenerated.

\section{Pitfalls of MRI Elastography}

The liver stiffness can increase due to various factors other than liver fibrosis, like in postprandial state, as the mesenteric circulation increases the blood flow to the portal system also increases, the normal liver being much more elastic does not cause much change in stiffness. But in the setting of fibrosis, the elasticity of the liver is reduced and hence shows a much higher value of stiffness. Inflammation of the liver can be challenging as it is difficult to distinguish from that of actual fibrosis. Alcohol being hepatotoxic, its intake can also increase stiffness by causing acute inflammation. Similar conditions like hepatic congestion, cholestasis, and infiltrative processes can increase liver stiffness $\left(\right.$ - Table 3). ${ }^{10}$

\section{Contraindications of MRI Elastography}

Main contraindications include non-MRI compatible implants and claustrophobia. Apart from these contraindications indicated in general for MRI, specific contraindications include iron overload and ascites. ${ }^{11}$ Iron interferes with the gradient sequences used, thereby interfering with image acquisition. The vibrations from the passive driver can be unbearable to some and accentuate claustrophobia. The newer sequences enable good results in cases of ascites also.

\section{Added Advantage}

As a part of the MRI sequences used, we often obtain T2weighted coronal and axial sections for comparison and it helps in the screening of the pancreas, gallbladder, and spleen often aiding the discovery of incidental lesions that may be missed in ultrasound.

\section{Fat Quantification of the Liver as an Addon}

Even though fat quantification is based on a completely different principle, it can be performed as an add on. Using a simple 40second sequence we can accurately estimate the percentage of fat and iron in the whole liver with the help of a completely automated process (-Fig. 11). These are based on principles of chemical shift imaging. There are even options to assess the fat and iron in any specific area manually. By quantifying fat and iron, it adds an extra dimension of chemical composition.

Table 3 Various pitfalls and causes

\begin{tabular}{|l|l|l|}
\hline Pitfall & Cause & Solution \\
\hline Post-prandial status & $\begin{array}{l}\text { Increase in portal circulation resulting in false } \\
\text { stiffness values, more prominent in diseased } \\
\text { liver. }\end{array}$ & Fasting of at least $4 \mathrm{~h}$ before the scan. \\
\hline Iron overload in liver & $\begin{array}{l}\text { Due to the paramagnetic properties of iron it } \\
\text { interferes with image acquisition. }\end{array}$ & $\begin{array}{l}\text { Good results if the scan is done post } \\
\text { phlebotomy. }\end{array}$ \\
\hline Inflammation & $\begin{array}{l}\text { Increased stiffness in hepatitis or direct injury } \\
\text { due to alcohol binging. }\end{array}$ & $\begin{array}{l}\text { Restrain from alcohol prior to the procedure } \\
\text { and compare lab parameters. }\end{array}$ \\
\hline Hepatic congestion & Reduces the elasticity. & $\begin{array}{l}\text { Specific peripheral pattern of increased } \\
\text { stiffness. }\end{array}$ \\
\hline
\end{tabular}




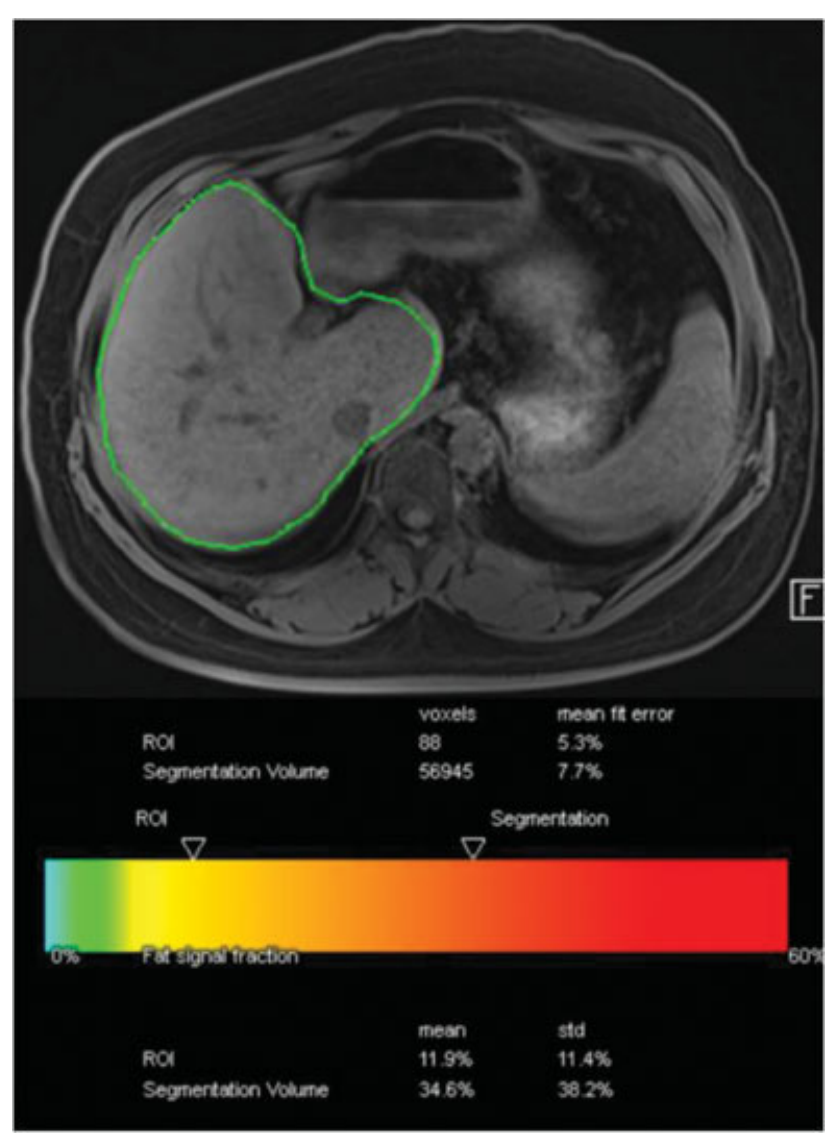

Fig. 11 The image shows fat quantification done on a 3-Tesla MRI with automatic segmentation of the whole liver and quantifying the fat percentage in the liver. In this patient 56,945 voxels of the liver were analyzed and showed a total of $34.6 \%$ fat.

In general, a myth persists that more the fat deposited in the liver more will be its stiffness. However, there are a number of studies stating there is no relation between the simple steatosis and fibrosis. ${ }^{22}$ Initial studies using ultrasound-based elastography techniques suggested an increase in stiffness of the liver corresponding to the fat deposit, now it is known to be caused by a phenomenon called dispersion. ${ }^{23}$ Even though fatty liver disease carries a risk of development of fibrosis in the future, it is not dependent on the amount of fat and rather on individual variability. Fibrosis only starts setting in when steatosis enters the stage of steatohepatitis.

\section{Recent Advances in Elastography}

The application of elastography is now being extended to various other deep-seated organs facilitated by MRI-based techniques and modified driver systems. Spleen stiffness assessed in combination with liver stiffness can give a much better prediction of portal hypertension as compared just spleen length. ${ }^{24}$ Renal elastography can also be done; early studies have shown decreased stiffness in cases of hepatorenal syndrome. ${ }^{25}$ Multi-frequency MRI techniques have led to the application in study of viscoelastic properties of the brain to better understand various degenerative conditions and better characterization of tumors. ${ }^{26}$ It also helps in better evaluation of tumors in the multiparametric approach of breast lesions. ${ }^{27}$
Early studies promise its use in assessing cardiac contractility, ${ }^{28}$ muscle tension, ${ }^{29}$ cervix, $^{30}$ uterus, $^{30}$ and pancreas. ${ }^{31}$

\section{Conclusion}

MRE has been FDA approved and is being used for detecting liver fibrosis since 2009, however, it is rarely being used in the Indian setting. It is a safe, noninvasive, and accurate technique to evaluate liver stiffness. It is superior in many aspects to ultrasound-based techniques and has certain advantages compared to biopsy. Considering the amount of population affected by liver diseases and advancement in treatment options, MRE can play an impactful role in early diagnosis, staging, and treatment response. MRI-based elastography techniques hold an exciting future in providing mechanical properties of tissues in various organs which are otherwise inaccessible by conventional methods.

Conflict of Interest

None declared.

\section{References}

1 Bedogni G, Nobili V, Tiribelli C. Epidemiology of fatty liver: an update. World J Gastroenterol 2014;20(27):9050-9054

2 Asrani SK, Devarbhavi H, Eaton J, Kamath PS. Burden of liver diseases in the world. J Hepatol 2019;70(01):151-171

3 Mariappan YK, Glaser KJ, Ehman RL. Magnetic resonance elastography: a review. Clin Anat 2010;23(05):497-511

4 Calzadilla Bertot L, Adams LA. The natural course of non-alcoholic fatty liver disease. Int J Mol Sci 2016;17(05):774

5 Serai SD, Obuchowski NA, Venkatesh SK, et al. Repeatability of MR elastography of liver: a meta-analysis. Radiology 2017;285(01): 92-100

6 Osna NA, Donohue TM Jr, Kharbanda KK. Alcoholic liver disease: pathogenesis and current management. Alcohol Res 2017;38(02): 147-161

7 Marra F, Lotersztajn S. Pathophysiology of NASH: perspectives for a targeted treatment. Curr Pharm Des 2013;19(29):5250-5269

8 Xu L, Gao PY. "Palpation by imaging": magnetic resonance elastography. Chin Med Sci J/Chung-kuo i hsueh k'o hsueh tsa chih 2006;21(04):281-286

9 Kennedy P, Wagner M, Castéra L, et al. Quantitative elastography methods in liver disease: current evidence and future directions. Radiology 2018;286(03):738-763

10 Guglielmo FF, Venkatesh SK, Mitchell DG, Liver MR. Liver MR elastography technique and image interpretation: pearls and pitfalls. Radiographics 2019;39(07):1983-2002

11 Srinivasa Babu A, Wells ML, Teytelboym OM, et al. Elastography in chronic liver disease: modalities, techniques, limitations, and future directions. Radiographics 2016;36(07):1987-2006

12 Venkatesh SK, Yin M, Ehman RL. Magnetic resonance elastography of liver: technique, analysis, and clinical applications. J Magn Reson Imaging 2013;37(03):544-555

13 Singh S, Venkatesh SK, Wang Z, et al. Diagnostic performance of magnetic resonance elastography in staging liver fibrosis: a systematic review and meta-analysis of individual participant data. Clin Gastroenterol Hepatol 2015;13(03):440-451.e6

14 Yin M, Talwalkar JA, Glaser KJ, et al. Assessment of hepatic fibrosis with magnetic resonance elastography. Clin Gastroenterol Hepatol 2007;5(10):1207-1213.e2

15 Wang Y, Ganger DR, Levitsky J, et al. Assessment of chronic hepatitis and fibrosis: comparison of MR elastography and diffusion-weighted imaging. AJR Am J Roentgenol 2011;196(03):553-561 
16 Rustogi R, Horowitz J, Harmath C, et al. Accuracy of MR elastography and anatomic MR imaging features in the diagnosis of severe hepatic fibrosis and cirrhosis. J Magn Reson Imaging 2012; 35(06):1356-1364

17 Low RN, Hassanein T. MR elastography: validation and reproducibility of measurements of mean liver stiffness and fibrosis. J Hepatol 2012;56:S415

18 Venkatesh SK, Wang G, Lim SG, Wee A. Magnetic resonance elastography for the detection and staging of liver fibrosis in chronic hepatitis B. Eur Radiol 2014;24(01):70-78

19 Ichikawa S, Motosugi U, Ichikawa T, et al. Magnetic resonance elastography for staging liver fibrosis in chronic hepatitis C. Magn Reson Med Sci 2012;11(04):291-297

20 Venkatesh SK, Yin M, Ehman RL. Magnetic resonance elastography of liver: clinical applications. J Comput Assist Tomogr 2013;37 (06):887-896

21 Low G, Kruse SA, Lomas DJ. General review of magnetic resonance elastography. World J Radiol 2016;8(01):59-72

22 Leitão HS, Doblas S, Garteiser P, et al. Hepatic fibrosis, inflammation, and steatosis: influence on the MR viscoelastic and diffusion parameters in patients with chronic liver disease. Radiology 2017;283(01):98-107

23 Barry CT, Mills B, Hah Z, et al. Shear wave dispersion measures liver steatosis. Ultrasound Med Biol 2012;38(02):175-182

24 Shin SU, Lee JM, Yu MH, et al. Prediction of esophageal varices in patients with cirrhosis: usefulness of three-dimensional MR elastography with echo-planar imaging technique. Radiology 2014;272(01):143-153

25 Low G, Owen NE, Joubert I, et al. Magnetic resonance elastography in the detection of hepatorenal syndrome in patients with cirrhosis and ascites. Eur Radiol 2015;25(10):2851-2858

26 Reiss-Zimmermann M, Streitberger KJ, Sack I, et al. High-resolution imaging of viscoelastic properties of intracranial tumours by multi-frequency magnetic resonance elastography. Clin Neuroradiol 2015;25(04):371-378

27 Siegmann KC, Xydeas T, Sinkus R, Kraemer B, Vogel U, Claussen CD. Diagnostic value of MR elastography in addition to contrastenhanced MR imaging of the breast-initial clinical results. Eur Radiol 2010;20(02):318-325

28 Kolipaka A, Aggarwal SR, McGee KP, et al. Magnetic resonance elastography as a method to estimate myocardial contractility. J Magn Reson Imaging 2012;36(01):120-127

29 Jenkyn TR, Ehman RL, An KN. Noninvasive muscle tension measurement using the novel technique of magnetic resonance elastography (MRE). J Biomech 2003;36(12):1917-1921

30 Jiang X, Asbach P, Streitberger KJ, et al. In vivo high-resolution magnetic resonance elastography of the uterine corpus and cervix. Eur Radiol 2014;24(12):3025-3033

31 Shi Y, Glaser KJ, Venkatesh SK, Ben-Abraham EI, Ehman RL. Feasibility of using 3D MR elastography to determine pancreatic stiffness in healthy volunteers. J Magn Reson Imaging 2015;41 (02):369-375 